\title{
Establishment and validation of a nomogram to predict the in-hospital death risk of nosocomial infections in cancer patients
}

\author{
Aimin Jiang ${ }^{1}$, Xin Shi ${ }^{2}$, Haoran Zheng ${ }^{1}$, Na Liu' ${ }^{1}$, Shu Chen ${ }^{3}$, Huan Gao ${ }^{1}$, Mengdi Ren ${ }^{1}$, Xiaogiang Zheng ${ }^{1}$, \\ Xiao Fu', Xuan Liang ${ }^{1}$, Zhiping Ruan ${ }^{1}$, Tao Tian $^{1 *}$ and Yu Yao ${ }^{1 *}$ (0)
}

\begin{abstract}
Background: Attributed to the immunosuppression caused by malignancy itself and its treatments, cancer patients are vulnerable to developing nosocomial infections. This study aimed to develop a nomogram to predict the in-hospital death risk of these patients.

Methods: This retrospective study was conducted at a medical center in Northwestern China. The univariate and multivariate logistic regression analyses were adopted to identify predictive factors for in-hospital mortality of nosocomial infections in cancer patients. A nomogram was developed to predict the in-hospital mortality of each patient, with receiver operating characteristic curves and calibration curves being generated to assess its predictive ability. Furthermore, decision curve analysis (DCA) was also performed to estimate the clinical utility of the nomogram.

Results: A total of 1,008 nosocomial infection episodes were recognized from 14,695 cancer patients. Extendedspectrum $\beta$-lactamase (ESBL)-producing Escherichia coli (15.5\%) was the most predominant causative pathogen. Besides, multidrug-resistant strains were discovered in $25.5 \%$ of cases. The multivariate analysis indicated that Eastern Cooperative Oncology Group Performance Status 3-4, mechanical ventilation, septic shock, hypoproteinemia, and length of antimicrobial treatment $<7$ days were correlated with higher in-hospital mortality. Patients who received curative surgery were correlated with favorable survival outcomes. Ultimately, a nomogram was constructed to predict the in-hospital mortality of nosocomial infections in cancer patients. The area under the curve values of the nomogram were 0.811 and 0.795 in the training and validation cohorts. The calibration curve showed high consistency between the actual and predicted in-hospital mortality. DCA indicated that the nomogram was of good clinical utility and more credible net clinical benefits in predicting in-hospital mortality.
\end{abstract}

Conclusions: Nosocomial infections stay conjoint in cancer patients, with gram-negative bacteria being the most frequent causative pathogens. We developed and verified a nomogram that could effectively predict the in-hospital death risk of nosocomial infections among these patients. Precise management of high-risk patients, early recognition of septic shock, rapid and adequate antimicrobial treatment, and dynamic monitoring of serum albumin levels may improve the prognosis of these individuals.

\footnotetext{
*Correspondence: tiantao0607@163.com;13572101611@163.com

1 Department of Medical Oncology, The First Affiliated Hospital of Xi'an

Jiaotong University, No. 277 Yanta West Road, Xi'an 710061, Shaanxi,

People's Republic of China

Full list of author information is available at the end of the article
} original author(s) and the source, provide a link to the Creative Commons licence, and indicate if changes were made. The images or other third party material in this article are included in the article's Creative Commons licence, unless indicated otherwise in a credit line to the material. If material is not included in the article's Creative Commons licence and your intended use is not permitted by statutory regulation or exceeds the permitted use, you will need to obtain permission directly from the copyright holder. To view a copy of this licence, visit http://creativecommons.org/licenses/by/4.0/. The Creative Commons Public Domain Dedication waiver (http://creativecommons.org/publicdomain/zero/1.0/) applies to the data made available in this article, unless otherwise stated in a credit line to the data. 
Keywords: Cancer patients, Nosocomial infections, Microbiological distribution, Antimicrobial susceptibility, Mortality

\section{Introduction}

Currently, malignant tumors have become one of the major public health problems that threaten human health worldwide. According to cancer statistics, in 2020, there will be 19.3 million newly diagnosed cancer cases and 10 million death cases all over the world [1]. No matter cancer incidence or mortality, solid tumors are at the forefront. Although significant advances in the treatment of malignancy have been made over the past few decades, the prognosis of these patients remains bleak. Besides, cancer patients are more vulnerable to developing severe infection owing to the malignancy itself and its treatments, especially surgery and cytotoxic therapies [2]. As a result, it delays the initiation of chemotherapy and reduces its relative dosage intensity (RDI). At the same time, it also prolongs hospitalization and increases the healthcare burden [3, 4]. Therefore, it is pivotal for clinicians to fully understand the local epidemiological and microbiological characteristics of nosocomial infections. It also can play an essential role in reducing the mortality of these individuals [2].

To date, a growing number of studies have focused on the bacteriological characteristics and antibiotic resistance pattern of nosocomial infections in patients with malignancy. However, most studies only concentrate on bloodstream infections (BSIs), despite other infections being more common than BSIs in these patients (such as respiratory tract and urinary tract infections). Furthermore, numerous studies reported that the microbial distribution and antibiotic sensitivity of cancer patients who developed nosocomial infections might be influenced by the source of infection $[5,6]$. Moreover, several previous studies have found that cancer patients with nosocomial infections were highly resistant to empirical antibiotic therapy due to the phenomenon of multidrug-resistant (MDR) [5, 7]. Importantly, although many studies have evaluated the clinical characteristics and prognostic factors of nosocomial infections in cancer patients, most studies enrolled a small number of sample size patients and drew inconclusive conclusions. Meanwhile, no study comprehensively investigated the clinical features of nosocomial infections in cancer patients and developed a predictive model to predict their in-hospital mortality. In this premier, we implemented this study to investigate the clinical features of nosocomial infections in patients with solid tumors and develop a nomogram to predict the in-hospital mortality of these patients.

\section{Methods and materials Study design}

This single-center retrospective cohort study was performed in the First Affiliated Hospital of Xi'an Jiaotong University, the medical center in Northwestern China. The oncology center in this hospital was composed of three departments (medical oncology ward, surgical oncology ward, and radiotherapy oncology ward). The electronic medical charts of all nosocomial infection cases hospitalized in the oncology center from August 2013 to May 2019 were retrospectively reviewed. All solid tumors were confirmed through histological or cytological pathology. All cancer patients identified with nosocomial infections during hospitalization were included in the present study if they were older than 18 years old. Patients under the age of 18 years old or without complete medical records were excluded. This research is approved by the local ethics committee of the First Affiliated Hospital of Xi'an Jiaotong University.

\section{Data collection}

Three investigators (AJ, HZ, and NL) extracted eligible patients' clinical data and laboratory parameters from electronic medical charts. The detailed clinical data were as follows: gender, age, smoking history, Eastern Cooperative Oncology Group performance status (ECOG-PS), the underlying malignancy type, stage of cancer, source of infection, comorbidities, the degree of fever, the types of anticancer treatment within one-month (surgery, chemotherapy, or radiotherapy), the regimen of empirical antibiotic treatment, length of antimicrobial therapy, the presence of indwelling catheters, the existence of septic shock during hospitalization, the use of a ventilator, and intensive care unit (ICU) admission during the hospitalization. Laboratory parameters of each patient were also reviewed, including the results of blood routine tests and serum albumin level. Furthermore, we collected the results of drug susceptibility tests of the isolated pathogens to commonly used antibiotics.

\section{Definitions}

Hospitalized cancer patients were considered as nosocomial infection cases if they met the following criteria [8]: (a) on the premise of excluding contamination of clinical specimens, the results of microbial culture indicated that at least one pathogen was positive ( $>48 \mathrm{~h}$ after hospital admission); (b) there were corresponding clinical manifestations, laboratory examination results, or radiological 
results recorded which is in electronic medical records; or (c) a clear infection type record that is acquired from the electronic medical records. The diagnosis of nosocomial respiratory tract infection was mainly based on microorganism culture from respiratory secretions, sputum, and or/ bronchoalveolar lavage fluid (BALF). Radiological findings of new or progressive infiltrate, consolidation, and effusion were also essential diagnostic conditions [8]. Besides, patients' symptoms, signs, and blood tests were also needed to be considered. The final diagnosis needs to be assessed by qualified physicians, and the radiologist was also needed to be consulted when necessary. Nosocomial urinary tract infection was considered by positive urine culture $\left(>10^{4}\right.$ colony-forming units $/ \mathrm{mL}$ of no more than two different species of microorganisms) obtained at least $48 \mathrm{~h}$ after hospital admission $[8,9]$. BSI was defined by a positive blood culture obtained from a patient at the time of hospital admission or within $48 \mathrm{~h}$ of admission, as previously described [10]. All BSIs cases in this study are hospital-acquired.

The shock was referred to as systolic blood pressure $<90 \mathrm{mmHg}$, and fluid therapy and/or vasoactive medications have no improvement on this condition. Fever was considered an axillary temperature of $38.3^{\circ} \mathrm{C}$ on one instance or a temperature of $>38.0{ }^{\circ} \mathrm{C}$ on two or more occasions throughout $12 \mathrm{~h}[11,12]$. Hypoproteinemia was defined as the level of serum albumin $<30 \mathrm{~g} / \mathrm{L}$.

MDR strains were defined based on the previous descriptions [13, 14]. Antimicrobial susceptibility testing was performed using the Kirby-Bauer disc diffusion method under the requirement of the Clinical and Laboratory Standards Institute (CLSI) guidelines [15].

\section{Study outcome and cohort establishment}

In this study, in-hospital mortality was used as the primary study outcome. Only death cases that were caused by nosocomial infections during hospitalization were selected for in-hospital mortality calculation. Nevertheless, death cases unrelated to nosocomial infections (such as malignancy itself or other complications) were not considered for in-hospital mortality calculation.

All eligible patients enrolled in the whole dataset were randomly divided into training and validation cohorts according to a ratio of 7:3 by exploiting the "createDataPartition" function in R software. The current study used the training cohort to develop a nomogram to predict the in-hospital death risk. The validation cohort was adopted to verify its predictive ability and clinical utility.

\section{Statistical analysis}

The continuous variables were summarized as means and standard deviations (SD) or medians and interquartile (IQR) as appropriate, while categorical variables were presented as frequency and percentage. The difference between categorical variables was compared using Chi-square or Fisher's exact tests, while a two-independent sample t-test or Mann-Whitney $\mathrm{U}$ test was used to compare the difference between continuous variables. Univariate and multivariate logistic regression analyses were adopted to investigate the independent risk factors for in-hospital mortality of nosocomial infections. Variables with a $P$ value $<0.05$ in the multivariate regression analysis were selected to construct a nomogram using $R$ software, "rms" and "regplot" packages to calculate each patient's in-hospital death risk. Furthermore, the receiver operating characteristic (ROC) curves and calibration curves were used to assess the predictive ability of the nomogram. Decision curve analysis (DCA) was also performed via R software, "DecisionCurve" package to estimate the clinical utility and net clinical benefits of the nomogram when it was adopted to support the clinical practice. All statistical analyses were conducted using the SPSS software version 22.0 and R software version 3.6.3.

\section{Results \\ Demographical characteristics of the participants}

During the study period, a total of 14,695 cancer patients were admitted to the oncology departments of the First Affiliated Hospital of Xi'an Jiaotong University. After excluding 13,612 cases of non-nosocomial infection, 10 cases without complete electronic medical records, and 65 cases diagnosed with benign disease, a total of 1,008 participants developed nosocomial infections during the period (Fig. 1). Among them, 563 were males (55.9\%) and 445 were females $(44.1 \%)$, with a median age of 61 years old (range: 54 to 68). Upper gastrointestinal tract tumor was the most common type of malignancy (285 cases, $28.3 \%$ ), followed by gynecological tumor (211 cases, 20.9\%) and lung cancer (193 cases, 19.1\%). Most of the patients (951 cases, 94.3\%) had ECOG-PS less than 3, and $603(59.8 \%)$ of the cases were diagnosed at an advanced stage (stage III-IV). Table 1 presented the detailed demographical characteristics of participants.

\section{Infection-related characteristics of the participants}

Subsequently, we also explored the infection-related characteristics of nosocomial infections in our study. Overall, 1008 (6.9\%) cancer patients developed nosocomial infections during the period. Respiratory tract infection, urinary tract infection, and BSI were the most common types of nosocomial infection, accounting for $37.3 \%, 24.8 \%$, and $12.9 \%$ of cases, respectively. In the current study, $84.1 \%$ of cases received empirical antibiotics therapy during hospitalization, with combination therapy being the most primary regimen (40.2\%), followed by $\beta$-lactam $/ \beta$-lactamase inhibitor (16.0\%), third-generation 


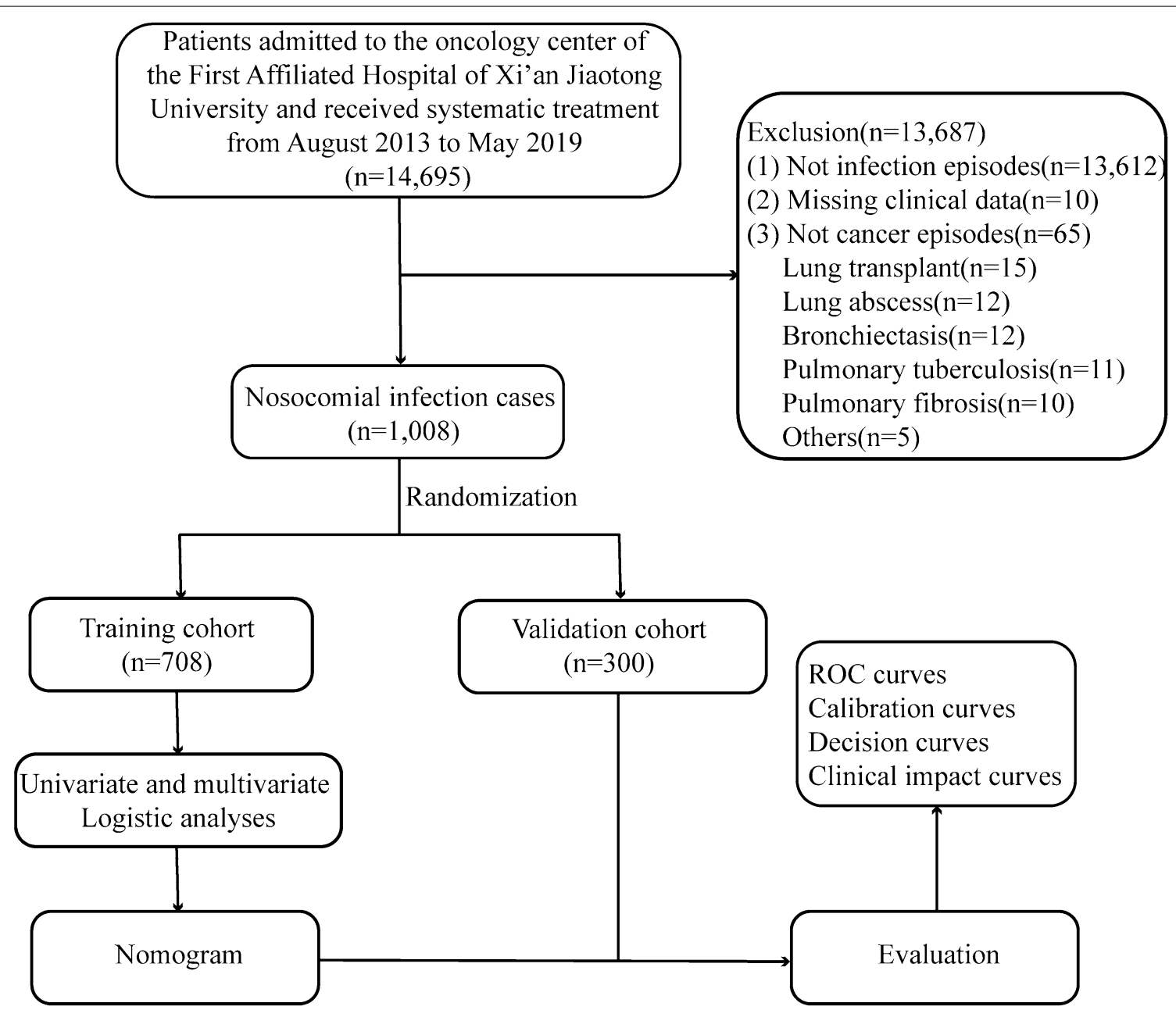

Fig. 1 Flow chart to investigate the clinical characteristics of nosocomial infections among cancer patients and establish a nomogram for in-hospital death risk prediction

cephalosporins (13.2\%), and fluoroquinolones (11.6\%). What's more, 99 patients $(9.8 \%)$ were admitted to the ICU during the study period, 66 patients $(6.5 \%)$ received mechanical ventilation, and 124 patients (12.3\%) experienced a septic shock (Table 1).

\section{Microbiological distribution characteristics and antimicrobial susceptibility analysis}

We further analyzed the microbiological distribution characteristics of nosocomial infections among patients with solid tumors. We found that gram-negative bacteria were the main causative pathogens of nosocomial infections, accounting for $63.3 \%$ of all pathogenic bacteria, followed by gram-positive cocci $(16.8 \%)$ and fungi (11.4\%). In our study, there were 257 cases $(25.5 \%)$ of nosocomial infections caused by MDR strains, with multi-drug resistant gram-negative bacilli (MDRGNB) being the most predominant pathogenic bacteria (24.8\%).
Overall, extended-spectrum $\beta$-lactamase (ESBL)-producing Escherichia coli (156 cases, 15.5\%), ESBL-negative E. coli (135 cases, 13.4\%), and Pseudomonas aeruginosa (86 cases, $8.5 \%$ ) were the most common pathogens that caused nosocomial infections in cancer patients, as summarized in Table 2. We also investigated the microbiological distribution features between different medical wards and different malignancy types. We observed that gram-negative bacteria were the most frequently isolated pathogens, no matter in different medical wards or malignancy types (Additional file 1: Fig. S1). Subsequently, we investigated the drug sensitivity of the isolated organisms to commonly used antibiotics. The results indicated that the isolated gram-negative bacilli exhibited high sensitivity to amikacin (95.0\%), meropenem (94.4\%), imipenem (93.4\%), and piperacillin/tazobactam (86.8\%) (Additional file 1: Fig. S2A), while the isolated gram-positive cocci exhibited high sensitivity to teicoplanin (100.0\%), 
Table 1 Demographic and clinical characteristics of nosocomial infections in cancer patients

\begin{tabular}{|c|c|c|c|c|c|c|}
\hline \multirow[t]{2}{*}{ Variables } & \multirow[t]{2}{*}{ Whole cohort $(n=1008)$} & \multicolumn{3}{|c|}{ Training cohort $(n=708)$} & \multirow{2}{*}{$\begin{array}{l}\text { Validation } \\
\text { cohort } \\
(n=300)\end{array}$} & \multirow[t]{2}{*}{$P^{b}$} \\
\hline & & Survival $(n=610)$ & Death $(n=98)$ & $P^{a}$ & & \\
\hline Sex (male) & $563(55.9)$ & $336(55.1)$ & $63(64.3)$ & 0.088 & $164(54.7)$ & 0.621 \\
\hline Age (years old) & $61(54-68)$ & $61(54-69)$ & $64(54-71)$ & 0.209 & $60(53-67)$ & $0.015^{*}$ \\
\hline$\geq 61$ & $533(52.9)$ & $332(54.4)$ & $60(61.2)$ & & $141(47.0)$ & \\
\hline$<61$ & $475(47.1)$ & $278(45.6)$ & $38(38.8)$ & & $159(53.0)$ & \\
\hline Tobacco use & $430(42.7)$ & $261(42.8)$ & $45(45.9)$ & 0.561 & $124(41.3)$ & 0.580 \\
\hline ECOG-PS & & & & $<0.001^{*}$ & & 0.992 \\
\hline $0-2$ & $951(94.3)$ & $591(96.9)$ & $77(78.6)$ & & $283(94.3)$ & \\
\hline $3-4$ & $57(5.7)$ & $19(3.1)$ & $21(21.4)$ & & $17(5.7)$ & \\
\hline \multicolumn{7}{|l|}{ Underlying malignancy type } \\
\hline Head and neck cancer & $34(3.4)$ & $16(2.6)$ & $8(8.2)$ & $0.012^{*}$ & $10(3.3)$ & 0.964 \\
\hline Lung cancer & $193(19.1)$ & $106(17.4)$ & $32(32.7)$ & $<0.001^{*}$ & $55(18.3)$ & 0.669 \\
\hline Upper gastrointestinal cancer & $285(28.3)$ & $180(29.5)$ & $23(23.5)$ & 0.220 & $82(27.3)$ & 0.666 \\
\hline Hepatobiliary and pancreatic cancer & $52(5.2)$ & $30(4.9)$ & $7(7.1)$ & 0.358 & $15(5.0)$ & 0.882 \\
\hline Breast cancer & $94(9.3)$ & $67(11.0)$ & $6(6.1)$ & 0.142 & $21(7.0)$ & 0.098 \\
\hline Colon and rectal cancer & $91(9.0)$ & $55(9.0)$ & $9(9.2)$ & 0.957 & $27(9.0)$ & 0.984 \\
\hline Genitourinary cancer & $48(4.8)$ & $33(5.4)$ & $3(3.1)$ & 0.463 & $12(4.0)$ & 0.460 \\
\hline Gynecological cancer & $211(20.9)$ & $123(20.2)$ & $10(10.2)$ & $0.019^{*}$ & $78(26.0)$ & $0.010^{*}$ \\
\hline Stage of cancer & & & & $0.045^{*}$ & & 0.729 \\
\hline Stage I-II & $405(40.2)$ & $252(41.3)$ & $30(30.6)$ & & $123(41.0)$ & \\
\hline Stage III-IV & $603(59.8)$ & $358(58.7)$ & $68(69.4)$ & & $177(59.0)$ & \\
\hline \multicolumn{7}{|l|}{ Comorbidities } \\
\hline COPD & $48(4.8)$ & $23(3.8)$ & $10(10.2)$ & $0.011^{*}$ & $15(5.0)$ & 0.817 \\
\hline Liver disease & $53(5.3)$ & $29(4.8)$ & $13(13.3)$ & $0.001^{*}$ & $11(3.7)$ & 0.141 \\
\hline Diabetes mellitus & $99(9.8)$ & $50(8.2)$ & $11(11.2)$ & 0.321 & $38(12.7)$ & $0.048^{*}$ \\
\hline Renal disease & $42(4.2)$ & $22(3.6)$ & $11(11.2)$ & $0.002^{*}$ & $9(3.0)$ & 0.228 \\
\hline \multicolumn{7}{|l|}{ Source of infection } \\
\hline Respiratory tract & $376(37.3)$ & $227(37.2)$ & $46(46.9)$ & 0.066 & $103(34.3)$ & 0.205 \\
\hline Gastrointestinal & $36(3.6)$ & $16(2.6)$ & $7(7.1)$ & $0.019^{*}$ & $13(4.3)$ & 0.396 \\
\hline Urinary tract & $250(24.8)$ & $159(26.1)$ & $13(13.3)$ & $0.006^{*}$ & $78(26.0)$ & 0.566 \\
\hline Skin and soft tissue & $98(9.7)$ & $69(11.3)$ & $2(2.0)$ & $0.005^{*}$ & $27(9.0)$ & 0.614 \\
\hline Thoracic cavity & $59(5.9)$ & $35(5.7)$ & $2(2.0)$ & 0.127 & $22(7.3)$ & 0.193 \\
\hline Abdominal cavity & $59(5.9)$ & $36(5.9)$ & $5(5.1)$ & 0.753 & $18(6.0)$ & 0.897 \\
\hline BSI & $130(12.9)$ & $68(11.1)$ & $23(23.5)$ & $0.001^{*}$ & $39(13.0)$ & 0.949 \\
\hline Existence of fever & $403(40.0)$ & $229(37.5)$ & $54(55.1)$ & $0.001^{*}$ & $120(40.0)$ & 0.993 \\
\hline Previous surgery (within 1 month) & & & & $<0.001^{*}$ & & 0.299 \\
\hline None & $668(66.3)$ & $387(63.4)$ & $87(88.8)$ & & $194(64.7)$ & \\
\hline Curative surgery & $314(31.2)$ & $205(33.6)$ & $8(8.2)$ & & $101(33.7)$ & \\
\hline Palliative surgery & $26(2.6)$ & $18(3.0)$ & $3(3.1)$ & & $5(1.7)$ & \\
\hline Previous chemotherapy (within 1 month) & & & & 0.245 & & 0.950 \\
\hline None & $625(62.0)$ & $371(60.8)$ & $70(71.4)$ & & $184(61.3)$ & \\
\hline Neoadjuvant & $12(1.2)$ & $8(1.3)$ & $1(1.0)$ & & $3(1.0)$ & \\
\hline Adjuvant & $198(19.6)$ & $126(20.7)$ & $14(14.3)$ & & $58(19.3)$ & \\
\hline 1st line & $114(11.3)$ & $71(11.6)$ & $6(6.1)$ & & $37(12.3)$ & \\
\hline 2nd line & $42(4.2)$ & $23(3.8)$ & $5(5.1)$ & & $14(4.7)$ & \\
\hline$\geq 3$ rd line & $17(1.7)$ & $11(1.8)$ & $2(2.0)$ & & $4(1.3)$ & \\
\hline Previous radiotherapy (within 1 month) & $142(14.1)$ & $89(14.6)$ & $9(9.2)$ & 0.150 & $44(14.7)$ & 0.731 \\
\hline \multicolumn{7}{|l|}{ Empirical antibiotic treatment } \\
\hline$\beta$-lactam $/ \beta$-lactamase inhibitor & $161(16.0)$ & $92(15.1)$ & $11(11.2)$ & 0.315 & $58(19.3)$ & 0.058 \\
\hline
\end{tabular}


Table 1 (continued)

\begin{tabular}{|c|c|c|c|c|c|c|}
\hline \multirow[t]{2}{*}{ Variables } & \multirow[t]{2}{*}{ Whole cohort $(n=1008)$} & \multicolumn{3}{|c|}{ Training cohort $(n=708)$} & \multirow{2}{*}{$\begin{array}{l}\text { Validation } \\
\text { cohort } \\
(n=300)\end{array}$} & \multirow[t]{2}{*}{$P^{b}$} \\
\hline & & Survival $(n=610)$ & Death $(n=98)$ & $P^{\mathrm{a}}$ & & \\
\hline Third-generation cephalosporins & $133(13.2)$ & $80(13.1)$ & $10(10.2)$ & 0.422 & $43(14.3)$ & 0.487 \\
\hline Carbapenems & $24(2.4)$ & $12(2.0)$ & $1(1.0)$ & 0.808 & $11(3.7)$ & 0.081 \\
\hline Fluoroquinolones & $117(11.6)$ & $71(11.6)$ & $9(9.2)$ & 0.476 & $37(12.3)$ & 0.639 \\
\hline Aminoglycosides & $8(0.8)$ & $3(0.5)$ & $0(0.0)$ & 1.000 & $5(1.7)$ & 0.100 \\
\hline Combination therapy & $405(40.2)$ & $250(41.0)$ & $55(56.1)$ & $0.005^{*}$ & $100(33.3)$ & $0.004^{*}$ \\
\hline Length of antimicrobial therapy (day) & $7(4-11)$ & $7(4-11)$ & $6(3-10)$ & $0.016^{*}$ & $7(4-10)$ & 0.546 \\
\hline$\geq 7$ & $563(56.4)$ & $359(50.7)$ & $45(6.4)$ & & $165(55.0)$ & \\
\hline$<7$ & $439(43.6)$ & $251(35.5)$ & $53(7.5)$ & & $135(45.0)$ & \\
\hline Presence of indwelling catheters & $564(56.0)$ & $350(57.4)$ & $45(45.9)$ & $0.034^{*}$ & $169(56.3)$ & 0.874 \\
\hline ICU admission & $99(9.8)$ & $68(11.1)$ & $13(13.3)$ & 0.541 & $18(6.0)$ & $0.008^{*}$ \\
\hline Mechanical ventilation & $66(6.5)$ & $35(5.7)$ & $14(14.3)$ & $0.002^{*}$ & $17(5.7)$ & 0.462 \\
\hline Septic shock & $124(12.3)$ & $58(9.5)$ & $37(37.8)$ & $<0.001^{*}$ & $29(9.7)$ & 0.097 \\
\hline \multicolumn{7}{|l|}{ Laboratory examination results } \\
\hline Hemoglobin (g/L; normal range 115-150) & $108.2 \pm 20.1$ & $108.9 \pm 19.5$ & $103.4 \pm 22.3$ & 0.010 & $108.3 \pm 20.56$ & 0.921 \\
\hline$<110$ & $536(53.2)$ & $325(53.3)$ & $61(62.2)$ & 0.098 & $150(50.0)$ & 0.189 \\
\hline $\begin{array}{l}\text { Platelet count }\left(\times 10^{9} / \mathrm{L} \text {; normal range }\right. \\
125-350)\end{array}$ & $211.1 \pm 113.4$ & $217.8 \pm 117.8$ & $172.9 \pm 113.7$ & $<0.001$ & $210.1 \pm 101.6$ & 0.852 \\
\hline$<100.0$ & $131(13.0)$ & $67(11.0)$ & $25(25.5)$ & $<0.001^{*}$ & $39(13.0)$ & 0.998 \\
\hline $\begin{array}{l}\text { White-cell count }\left(\times 10^{9} / \mathrm{L} \text {; normal range }\right. \\
4.0-10.0)\end{array}$ & $8.1 \pm 5.1$ & $8.2 \pm 5.1$ & $9.1 \pm 6.8$ & 0.257 & $7.6 \pm 4.2$ & $0.021^{*}$ \\
\hline$>10.0$ & $265(26.3)$ & $168(27.5)$ & $34(34.7)$ & 0.146 & $63(21.0)$ & $0.013^{*}$ \\
\hline$<4.0$ & $161(16.0)$ & $93(15.2)$ & $20(20.4)$ & 0.195 & $48(16.0)$ & 0.987 \\
\hline Neutrophils count $\left(\times 10^{9} / \mathrm{L}\right)$ & $6.6 \pm 4.8$ & $6.6 \pm 4.9$ & $7.7 \pm 6.4$ & 0.107 & $6.1 \pm 4.0$ & $0.026^{*}$ \\
\hline $\begin{array}{l}\text { Lymphocytes count }\left(\times 10^{9} / \mathrm{L} \text {; normal range }\right. \\
1.1-3.2)\end{array}$ & $1.0 \pm 0.6$ & $1.0 \pm 0.6$ & $0.8 \pm 0.6$ & 0.009 & $1.0 \pm 0.6$ & 0.800 \\
\hline$<1.0$ & $583(57.8)$ & $352(57.7)$ & $71(72.4)$ & $0.006^{*}$ & $160(53.3)$ & 0.059 \\
\hline Albumin (g/L; normal range 40-55) & $34.2 \pm 6.1$ & $34.6 \pm 5.8$ & $30.9 \pm 6.1$ & $<0.001$ & $34.5 \pm 6.2$ & 0.303 \\
\hline$<30.0$ & $263(26.1)$ & $140(23.0)$ & $49(50.0)$ & $<0.001^{*}$ & $74(24.7)$ & 0.503 \\
\hline
\end{tabular}

ECOG-PS, Eastern Cooperative Oncology Group Performance Status; COPD, chronic obstructive pulmonary disease; BSI, bloodstream infection; ICU, intensive care unit

a $P$ value for univariate analysis of the training cohort

${ }^{b} P$ value for clinical characteristics analysis between the training cohort and validation cohort

${ }^{*}$ Indicates statistical significance

vancomycin (100.0\%), linezolid (100.0\%), and tigecycline (100.0\%) (Additional file 1: Fig. S2B).

\section{Predictive factors for in-hospital death risk of nosocomial infections in cancer patients}

The overall case-fatality rate is $12.1 \%$ of nosocomial infections among cancer patients in our study. We compared the clinical characteristics, infection-related characteristics, and the distribution of causative pathogens of patients based on the survival outcomes during hospitalization in the training cohort to identify potential predictive factors of in-hospital mortality. The univariate logistic regression analysis indicated that several factors were correlated with the in-hospital mortality of nosocomial infections in these patients $(P<0.05$, Tables 1 and 2). Subsequently, we performed multivariate analysis to identify the independent predictive factors of in-hospital death risk. Ultimately, we observed that ECOG-PS 3-4 [odds ratio (OR): 4.46, 95\% confidence interval (CI) 2.04-9.74; $P<0.001]$, previous curative surgery (OR $0.18,95 \%$ CI $0.07-0.52 ; P=0.001$ ), length of antimicrobial therapy $<7$ days (OR 2.54, 95\% CI 1.40-4.60; $P=0.002)$, mechanical ventilation (OR 4.17, 95\% CI 1.42-12.23; $P=0.009$ ), septic shock (OR 3.16 , 95\% CI $1.37-7.30 ; P=0.007)$, and hypoproteinemia (OR 2.34, 95\% CI 1.34-4.10; $P=0.003$ ) were independent predictive factors of in-hospital death risk (Table 3). 
Table 2 Causative pathogens of all nosocomial infection episodes in cancer patients

\begin{tabular}{|c|c|c|c|c|c|c|}
\hline \multirow[t]{2}{*}{ Causative organisms } & \multirow{2}{*}{$\begin{array}{l}\text { Whole cohort } \\
(n=1008)\end{array}$} & \multicolumn{3}{|c|}{ Training cohort $(n=708)$} & \multirow{2}{*}{$\begin{array}{l}\text { Validation } \\
\text { cohort }(n=300)\end{array}$} & \multirow[t]{2}{*}{$P^{b}$} \\
\hline & & Survival $(n=610)$ & Death $(n=98)$ & $P^{a}$ & & \\
\hline Gram-negative bacteria & $388(38.5)$ & $229(37.5)$ & $31(31.6)$ & 0.260 & $128(42.7)$ & 0.076 \\
\hline Escherichia coli & $135(13.4)$ & $71(11.6)$ & $10(10.2)$ & 0.679 & $54(18.0)$ & $0.005^{*}$ \\
\hline Klebsiella pneumoniae & $65(6.4)$ & $38(6.2)$ & $11(11.2)$ & 0.071 & $16(5.3)$ & 0.348 \\
\hline Pseudomonas aeruginosa & $86(8.5)$ & $54(8.9)$ & $4(4.1)$ & 0.110 & $28(9.3)$ & 0.553 \\
\hline Enterobacter spp. & $34(3.4)$ & $21(3.4)$ & $2(2.0)$ & 0.675 & $11(3.7)$ & 0.737 \\
\hline Klebsiella oxytoca & $4(0.4)$ & $3(0.5)$ & $0(0.0)$ & 1.000 & $1(0.3)$ & 1.000 \\
\hline Proteus mirabilis & $13(1.3)$ & $8(1.3)$ & $2(2.0)$ & 0.915 & $3(1.0)$ & 0.822 \\
\hline Salmonella enterica serovar & $2(0.2)$ & $1(0.2)$ & $0(0.0)$ & 1.000 & $1(0.3)$ & 0.507 \\
\hline Haemophilus spp & $42(4.2)$ & $28(4.6)$ & $2(2.0)$ & 0.372 & $12(4.0)$ & 0.863 \\
\hline Serratia & $7(0.7)$ & $5(0.8)$ & $0(0.0)$ & 1.000 & $2(0.7)$ & 1.000 \\
\hline Gram-positive bacteria & $143(14.2)$ & $87(14.3)$ & $14(14.3)$ & 0.995 & $42(14.0)$ & 0.912 \\
\hline Staphylococcus aureus & $71(7.0)$ & $40(6.6)$ & $7(7.1)$ & 0.829 & $24(8.0)$ & 0.440 \\
\hline MRSA & $7(0.7)$ & $4(0.7)$ & $2(2.0)$ & 0.196 & $1(0.3)$ & 0.681 \\
\hline Streptococcus pneumoniae & $37(3.7)$ & $21(3.4)$ & $3(3.1)$ & 1.000 & $13(4.3)$ & 0.466 \\
\hline Coagulase-negative staphylococci & $15(1.5)$ & $13(2.1)$ & $1(1.0)$ & 0.732 & $1(0.3)$ & 0.092 \\
\hline Streptococcus anginosus & $13(1.3)$ & $9(1.5)$ & $1(1.0)$ & 1.000 & $3(1.0)$ & 0.822 \\
\hline Enterococcus & $26(2.6)$ & $15(2.5)$ & $6(6.1)$ & 0.096 & $5(1.7)$ & 0.234 \\
\hline E. faecalis & $12(1.2)$ & $7(1.1)$ & $3(3.1)$ & 0.303 & $2(0.7)$ & 0.496 \\
\hline E. faecium & $10(1.0)$ & $5(0.8)$ & $2(2.0)$ & 0.251 & $3(1.0)$ & 1.000 \\
\hline Enterococcus spp & $4(0.4)$ & $3(0.5)$ & $1(1.0)$ & 0.450 & $0(0.0)$ & 0.324 \\
\hline Anaerobes & $6(0.6)$ & $5(0.8)$ & $0(0.0)$ & 1.000 & $1(0.3)$ & 0.676 \\
\hline Fungi & $115(11.4)$ & $60(9.8)$ & $21(21.4)$ & 0.001 & $34(11.3)$ & 0.961 \\
\hline Candida albicans & $79(7.8)$ & $44(7.2)$ & $13(13.3)$ & $0.041^{*}$ & $22(7.3)$ & 0.698 \\
\hline Candida spp. & $14(1.4)$ & $7(1.1)$ & $2(2.0)$ & 0.805 & $5(1.7)$ & 0.844 \\
\hline Aspergillus flavus & $22(2.2)$ & $9(1.5)$ & $6(6.1)$ & $0.010^{*}$ & $7(2.3)$ & 0.831 \\
\hline MDRGNB & $250(24.8)$ & $162(26.6)$ & $23(23.5)$ & 0.518 & $65(21.7)$ & 0.134 \\
\hline ESBL-producing Escherichia coli & $156(15.5)$ & $102(16.7)$ & $17(17.3)$ & 0.878 & $37(12.3)$ & 0.073 \\
\hline MDR Pseudomonas aeruginosa & $13(1.3)$ & $10(1.6)$ & $0(0.0)$ & 0.415 & $3(1.0)$ & 0.822 \\
\hline Acinetobacter baumannii & $30(3.0)$ & $18(3.0)$ & $1(1.0)$ & 0.447 & $11(3.7)$ & 0.401 \\
\hline Stenotrophomonas maltophilia & $16(1.6)$ & $8(1.3)$ & $3(3.1)$ & 0.390 & $5(1.7)$ & 1.000 \\
\hline ESBL-producing Klebsiella pneumoniae & $31(3.1)$ & $20(3.3)$ & $2(2.0)$ & 0.732 & $9(3.0)$ & 0.928 \\
\hline Carbapenem-resistant Enterobacteriaceae & $4(0.4)$ & $4(0.7)$ & $0(0.0)$ & 1.000 & $0(0.0)$ & 0.324 \\
\hline Polymicrobial & $80(7.9)$ & $52(8.5)$ & $3(3.1)$ & 0.061 & $25(8.3)$ & 0.762 \\
\hline
\end{tabular}

MRSA, Methicillin-resistant S. aureus; MDRGNB, multidrug-resistant gram-negative bacilli; ESBL, extended-spectrum $\beta$-lactamase; MDR: multidrug-resistant

a $P$ value for univariate analysis of the training cohort

${ }^{b} P$ value for clinical characteristics analysis between the training cohort and validation cohort

* Indicates statistical significance

\section{Construction and validation of the nomogram}

A nomogram was constructed based on the six independent predictive variables to estimate each patient's in-hospital mortality easily. The clinicians could evaluate the in-hospital death risk of cancer patients once they developed nosocomial infections during hospitalization. Furthermore, they could recognize high-risk patients and take appropriate measures to minimize the in-hospital death risk. Figure 2 gave an example to show how the nomogram could be used as an accurate prognostic stratification tool for nosocomial infections in cancer patients. Additionally, multiple tests were performed to assess the predictive ability and clinical utility of the nomogram in predicting the in-hospital death risk of nosocomial infections among oncological patients in the training and validation cohorts. We observed that the area under the curve (AUC) values of the nomogram were 0.811 (95\% CI 0.765 to 0.857$)$ and 
Table 3 Multivariate logistic regression analysis for prognostic factors of nosocomial infections in cancer patients in the training cohort

\begin{tabular}{|c|c|c|c|c|c|}
\hline Variables & $\beta$ & SE & Wald & OR $(95 \% \mathrm{Cl})$ & $P$ \\
\hline \multicolumn{6}{|l|}{ ECOG-PS } \\
\hline \multicolumn{6}{|l|}{$0-2$} \\
\hline $3-4$ & 1.49 & 0.40 & 14.02 & $4.46(2.04-9.74)$ & $<0.001^{*}$ \\
\hline \multicolumn{6}{|l|}{ Underlying malignancy type } \\
\hline Head and neck cancer & 0.85 & 0.56 & 2.30 & $2.33(0.78-6.98)$ & 0.130 \\
\hline Lung cancer & 0.26 & 0.33 & 0.62 & $1.30(0.68-2.48)$ & 0.431 \\
\hline Gynecological cancer & -0.60 & 0.43 & 1.94 & $0.55(0.24-1.28)$ & 0.164 \\
\hline Stage of cancer & -0.03 & 0.29 & 0.01 & $0.97(0.55-1.71)$ & 0.924 \\
\hline \multicolumn{6}{|l|}{ Source of infection } \\
\hline Gastrointestinal tract & 0.13 & 0.58 & 0.05 & $1.14(0.36-3.59)$ & 0.819 \\
\hline Urinary tract & -0.54 & 0.42 & 1.63 & $0.59(0.26-1.33)$ & 0.202 \\
\hline Skin and soft tissue & -1.25 & 0.79 & 2.50 & $0.29(0.06-1.35)$ & 0.114 \\
\hline BSI & -0.66 & 0.46 & 2.06 & $0.52(0.21-1.27)$ & 0.152 \\
\hline \multicolumn{6}{|l|}{ Comorbidities } \\
\hline COPD & 0.14 & 0.54 & 0.06 & $1.15(0.40-3.32)$ & 0.803 \\
\hline Liver disease & 0.80 & 0.44 & 3.39 & $2.23(0.95-5.22)$ & 0.066 \\
\hline Renal disease & 0.86 & 0.50 & 2.95 & $2.36(0.89-6.26)$ & 0.086 \\
\hline Existence of fever & 0.27 & 0.32 & 0.70 & $1.31(0.70-2.45)$ & 0.405 \\
\hline Previous surgery (within 1 month) & & & & & $0.006^{*}$ \\
\hline None & & & 10.21 & & \\
\hline Curative surgery & -1.69 & 0.53 & 10.20 & $0.18(0.07-0.52)$ & $0.001^{*}$ \\
\hline Palliative surgery & -0.46 & 0.77 & 0.36 & $0.63(0.14-2.87)$ & 0.550 \\
\hline \multicolumn{6}{|c|}{ Empirical antibiotic treatment (within 1 month) } \\
\hline Combination therapy & 0.36 & 0.32 & 1.28 & $1.43(0.77-2.67)$ & 0.258 \\
\hline Length of antimicrobial therapy $<7$ days & 0.93 & 0.30 & 9.39 & $2.54(1.40-4.60)$ & $0.002^{*}$ \\
\hline Presence of indwelling catheters & -0.16 & 0.29 & 0.31 & $0.85(0.48-1.51)$ & 0.576 \\
\hline Mechanical ventilation & 1.43 & 0.55 & 6.78 & $4.17(1.42-12.23)$ & $0.009^{*}$ \\
\hline Septic shock & 1.15 & 0.43 & 7.30 & $3.16(1.37-7.30)$ & $0.007^{*}$ \\
\hline \multicolumn{6}{|l|}{ Laboratory examination results } \\
\hline Platelet count $<100.0 \times 10^{9} / \mathrm{L}$ & 0.19 & 0.34 & 0.31 & $1.21(0.62-2.36)$ & 0.578 \\
\hline Lymphocytes count $<1.0 \times 10^{9} / \mathrm{L}$ & 0.33 & 0.29 & 1.30 & $1.40(0.79-2.48)$ & 0.255 \\
\hline Albumin $<30.0 \mathrm{~g} / \mathrm{L}$ & 0.85 & 0.29 & 8.84 & $2.34(1.34-4.10)$ & $0.003^{*}$ \\
\hline \multicolumn{6}{|l|}{ Fungi } \\
\hline Candida albicans & 0.22 & 0.43 & 0.25 & $1.24(0.53-2.90)$ & 0.617 \\
\hline Aspergillus flavus & 0.64 & 0.69 & 0.85 & $1.89(0.49-7.32)$ & 0.355 \\
\hline
\end{tabular}

SE, standardized error; OR, odds ratio; ECOG-PS, Eastern Cooperative Oncology Group Performance Status; BSI, bloodstream infection; COPD, chronic obstructive pulmonary disease

${ }^{*}$ Indicates statistical significance

0.795 (95\% CI 0.745 to 0.846$)$ in the training and validation cohorts (Fig. 3A, B). Besides, the calibration curves also revealed high consistencies between the actual and predicted in-hospital mortality in the two cohorts (Fig. 3C, D), suggesting that the constructed nomogram had good discrimination and calibration abilities. Given that the ROC curves and calibration curves are based on the specificity and sensitivity of the predictive model, they could not recognize "false positive" and "false negative" cases. DCA was conducted to evaluate the clinical utility and net clinical benefits of the nomogram when it was adopted to support clinical practice. We found higher clinical net benefits when the risk threshold was between 0 and 1.0 (Fig. 3E, F), indicating that the nomogram could significantly reduce the inhospital death risk of nosocomial infections in cancer patients when used to guide clinical practice. Consistent results were also observed in clinical impact curves 


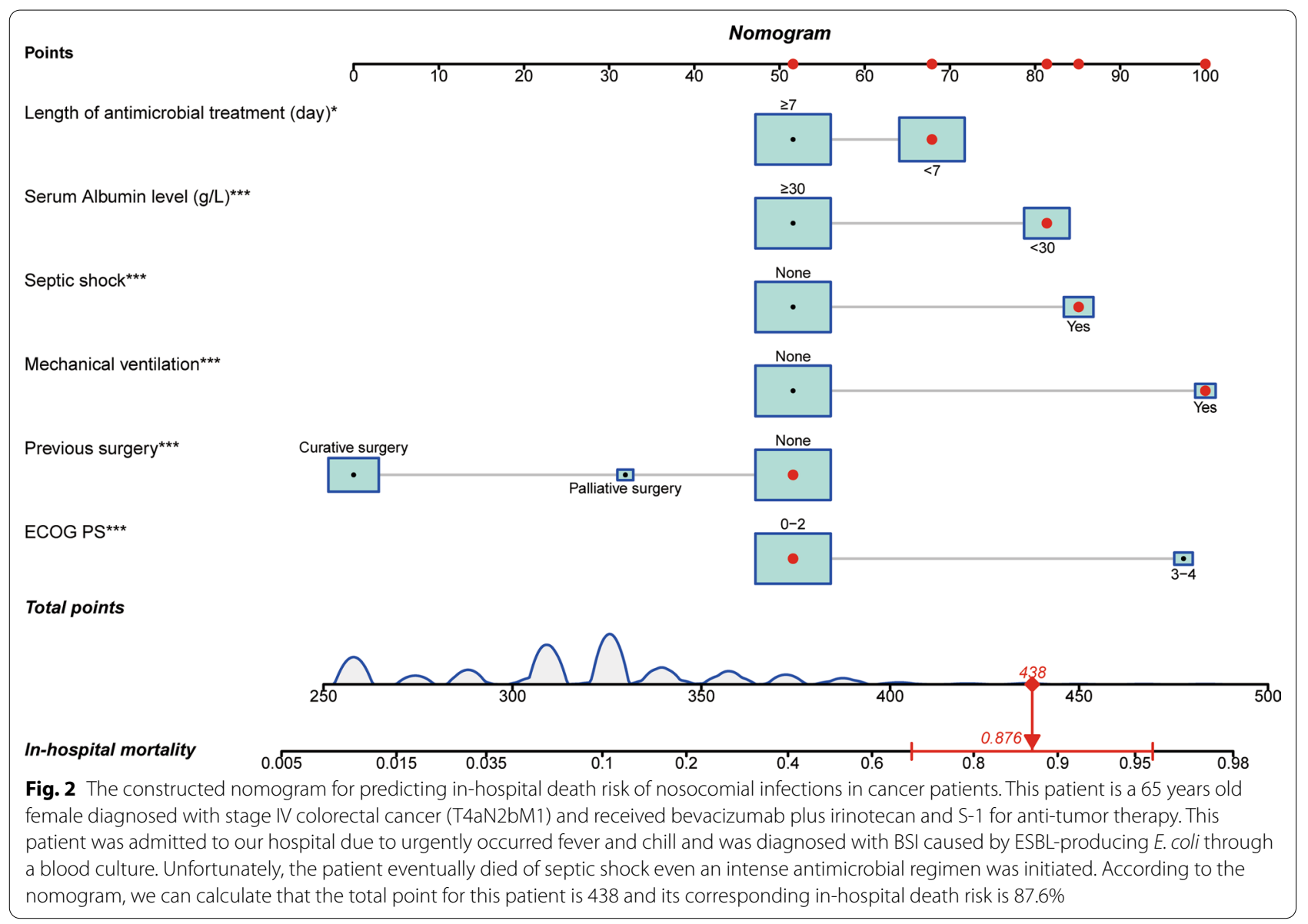

both in training and validation cohorts (Additional file 1: Fig. S3).

\section{Discussion}

Patients with malignancy are more vulnerable to developing infections for various reasons (e.g., malnutrition, invasive procedures, surgery, immune suppression caused by cytotoxic treatment) [2]. Cancer patients have a bleak prognosis in nosocomial infections than noncancer patients, which means poor outcomes, prolonged hospitalization, more ICU admissions, extra medical costs, and higher mortality [3, 4, 16, 17]. Therefore, we conducted the current study to fully understand the clinical characteristics of nosocomial infections in patients with solid tumors and established a predictive model to predict the in-hospital mortality of these patients. This study systematically explored the clinical features of nosocomial infections in cancer patients, including microbiological distribution characteristics, infectionrelated features, and antimicrobial resistance patterns. Most importantly, we investigated the predictive factors for in-hospital death risk of nosocomial infections in cancer patients and developed and verified a nomogram that could accurately estimate the in-hospital death risk for each patient.

In the current study, $6.9 \%$ of patients with malignancy had nosocomial infections over the six years study period, which was low compared with the results of studies conducted in Ethiopia (19.4\%) [6], Sudan (48.1\%) [18], and India (31.33\%) [19]. This discrepancy could be explained by the fact that the prevalence of hospitalacquired infections in cancer patients varies widely from region to region. In our study, we found that gram-negative bacteria were responsible for $63.3 \%$ of nosocomial infection episodes. In contrast, gram-positive cocci were responsible for $16.8 \%$ of cases, consistent with the results in other hospital settings [2, 20, 21]. However, several studies reported that gram-positive cocci represented the primary pathogens for nosocomial infections [6, 22-24]. This may be attributed to the population in these studies including hematological patients, and most of them combined with neutropenia. Furthermore, the epidemiology features of nosocomial infections in oncological patients have swerved from gram-positive cocci to gram-negative bacteria in the previous 20 years, as we saw worldwide [25]. These findings suggest that regular monitoring is 


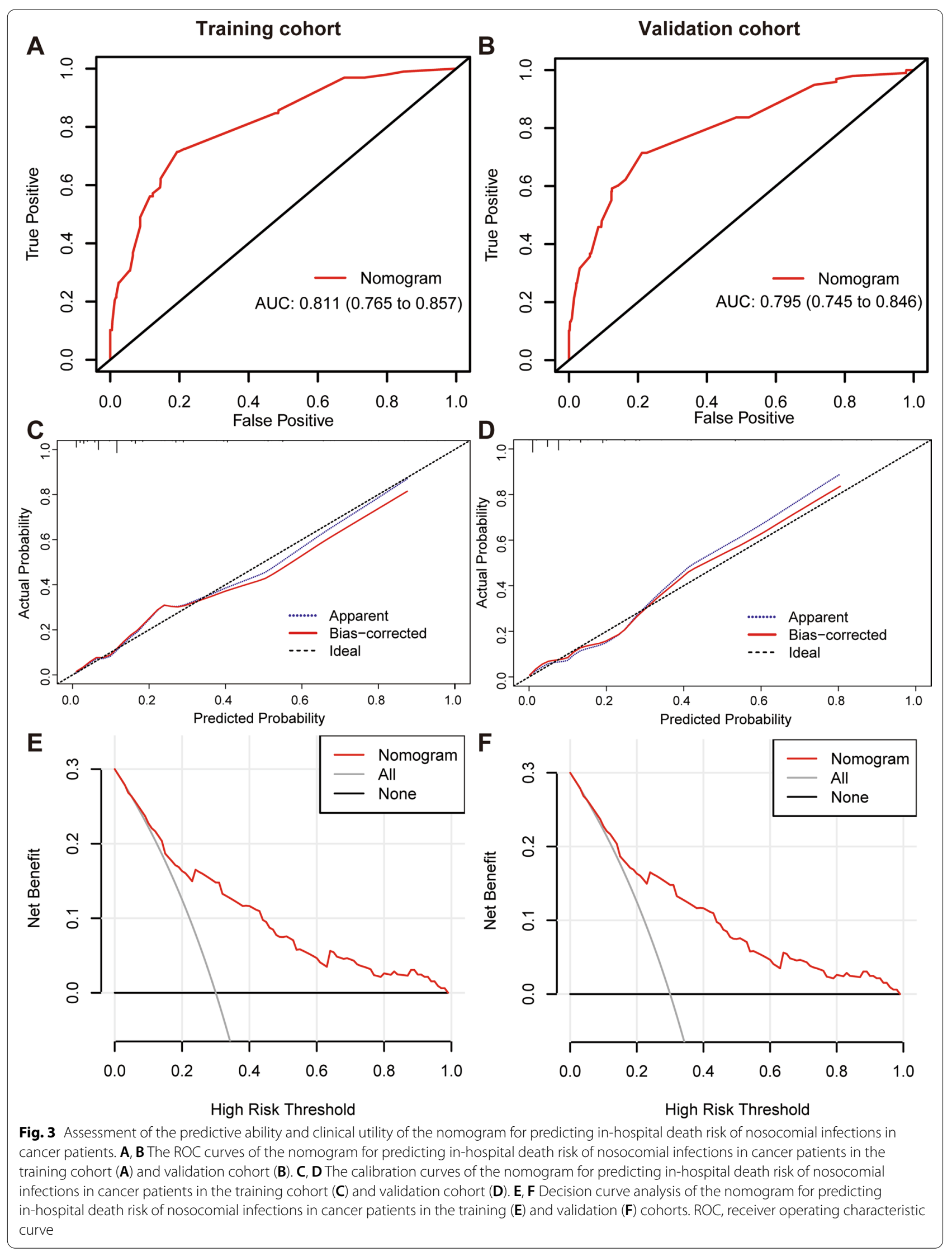


necessary to understand the epidemiological characteristics of nosocomial infections among cancer patients in the local hospital.

In this study, the MDR phenomenon was observed in $25.5 \%$ of nosocomial infection cases, with MDRGNB being the most predominant causative pathogens, accounting for $24.8 \%$ of all infection episodes. Of these, ESBL-producing $E$. coli was the most common MDR pathogen. A similar result was also reported in a retrospective study conducted in Spain [26]. Recently, ESBLproducing $E$. coli has emerged as an important pathogen associated with nosocomial infections worldwide. ESBL has been a crucial mechanism of third-generation cephalosporin resistance, leading to a series of treatment problems [27]. Therefore, it is critical for cancer patients with nosocomial infections to initiate appropriate antibiotic treatment. Generally, the $\beta$-lactam $+\beta$-lactamase inhibitor is the most common empirical antibiotic treatment in cancer patients with hospital-acquired infections. However, the antimicrobial susceptibility of the isolated gramnegative bacteria showed that these pathogens exhibited extraordinary sensitivity to piperacillin/tazobactam, meropenem, imipenem, and amikacin, regardless of their ESBL status, which is in line with a study conducted in southern Taiwan [21]. Thus, piperacillin/tazobactam could serve as an initial empirical antibiotic regimen for cancer patients with nosocomial infections.

In the current study, $12.1 \%$ of cancer patients died from nosocomial infections, which is lower than the results of previous studies reported by Marin et al. (32\%) [28] and Wisplinghoff et al. (35.5\%) [24]. This difference can be explained by the fact that BSI was the most common infection type in these studies. Additionally, we investigated the predictive factors for in-hospital death risk of nosocomial infections among cancer patients. We identified that ECOG-PS 3-4, mechanical ventilation, septic shock, inadequate antimicrobial treatment, and hypoproteinemia were independent risk factors for inhospital death risk. However, previous curative surgery was a protective factor for in-hospital mortality. Interestingly, Tu et al. [29] reported that intra-abdominal infection did not cause reduced long-term survival of patients who went through curative surgery. On the one hand, it may be attributed to the fact that patients who received curative surgery had no distant metastasis. On the other hand, considering only a small number of patients were enrolled in this subgroup, it needs to be further validated before drawing the conclusion.

Generally speaking, cancer patients with worse ECOGPS were correlated with unfavorable survival outcomes. In this study, we observed that patients with worse ECOG-PS had higher in-hospital mortality when these patients developed nosocomial infections. It suggests that we should pay close attention to these populations. Besides, appropriate and precise management should be initiated rapidly once infection onsets. We also found that patients who received mechanical ventilation and inadequate antimicrobial therapy during hospitalization were associated with a worse prognosis, which was compatible with previous studies [11, 20, 29-31]. Septic shock was identified as the most predominant risk factor for a worse survival probability of nosocomial infections [11, 20, 32]. As we know, patients with septic shock or who received mechanical ventilation always combined with circulatory and/ or respiratory dysfunction, resulting in poor clinical outcomes. Furthermore, we observed that patients with hypoproteinemia were significantly related to higher in-hospital mortality. Previous studies reported that hypoproteinemia was frequently related to reduced quality of life and diminished life expectancy due to immunosuppression and diminished muscle mass in patients with malignancy [33, 34]. In addition, Paccagnella $\mathrm{A}$ et al. also reported that hypoproteinemia in cancer patients could result in malnutrition and weight loss, leading to a poor prognosis and increased cancer-associated deaths in these patients [35, 36]. Taken together, clinicians should focus more on the respiratory and circulatory conditions of cancer patients once they developed severe nosocomial infections. Besides, it also suggested that rapid recognition of septic shock, early and effective empirical antibiotics treatment, monitoring of nutritional status, and supportive care are also crucial in improving the prognosis of these patients.

Currently, nomogram is widely used in the research of cancer and other fields. The nomogram can transform the sophisticated regression equation into an intuitive graph, making the patients' prediction risk readable. In the present study, we developed a nomogram to predict the in-hospital death risk of nosocomial infections in cancer patients. We also evaluated its predictive ability and clinical utility when it was adopted to support decision-making in practice. Altogether, the nomogram has good performance in predicting the in-hospital death risk of these individuals. To the best of our knowledge, this is the first study that systematically evaluated the clinical features of nosocomial infections among cancer patients in Northwestern China. Most importantly, we developed and verified a nomogram that could accurately predict the in-hospital death risk of nosocomial infections among these patients. Nevertheless, our study also has several inevitable shortcomings. First, it is challenging to collect some variables (such as concrete chemotherapeutic and radioactivity dosage, concrete antibiotics treatment information before admission, and some detailed laboratory examination results) because of the design of retrospective analysis. Thus, potential biases might exist in 
this study. Second, although we established a nomogram that could effectively predict the in-hospital death risk of nosocomial infections in patients with solid tumors and validated its predictive ability in a validation cohort, lacking an independent external validation cohort is a disadvantage for this study. Therefore, multicenter retrospective and well-designed prospective studies are urgently needed in the future to verify the performance of the nomogram.

\section{Conclusions}

In conclusion, nosocomial infections are common in oncological patients. Gram-negative bacteria are still the most frequently isolated pathogens. MDR phenomenon is not rare for nosocomial infections in these patients, with MDRGNB being the most predominant MDR strain. Besides, we constructed and validated a novel nomogram that could accurately predict the in-hospital death risk of nosocomial infections in cancer patients. Precise management of high-risk patients, early recognition of septic shock, rapid and adequate antimicrobial treatment, and dynamic monitoring of serum albumin levels may improve the prognosis of these individuals.

\begin{abstract}
Abbreviations
RDI: Relative dosage intensity; BSIs: Bloodstream infections; MDR: Multidrugresistant; ECOG-PS: Eastern Cooperative Oncology Group performance status; ICU: Intensive care unit; BALF: Bronchoalveolar lavage fluid; CLSI: Clinical and Laboratory Standards Institute; SD: Standard deviations; IQR: Interquartile; ROC: Receiver operating characteristic; DCA: Decision curve analysis; COPD: Chronic obstructive pulmonary disease; MDRGNB: Multi-drug resistant gramnegative bacilli; ESBL: Extended-spectrum $\beta$-lactamase; OR: Odds ratio; Cl: Confidence interval; AUC: Area under the curve; TZP: Piperacillin/tazobactam; SXT: Sulfamethoxazole-trimethoprim.
\end{abstract}

\section{Supplementary Information}

The online version contains supplementary material available at https://doi. org/10.1186/s13756-022-01073-3.

Additional file 1: Fig. S1. Microbiological distribution characteristics of nosocomial infections in cancer patients. (A) Microbiological distribution characteristics between different medical wards. (B) Microbiological distribution characteristics between different cancer types. Fig. S2. Antimicrobial resistance patterns of nosocomial infections in cancer patients. (A) Antimicrobial resistance pattern of gram-negative bacilli. (B) Antimicrobial resistance pattern of gram-positive cocci. TZP, Piperacillin/tazobactam; SXT, Sulfamethoxazole-trimethoprim. Fig. S3. Clinical impact curves of the nomogram for predicting in-hospital death risk of nosocomial infections in cancer patients in the training $(\mathbf{A})$ and validation (B) cohorts.

\section{Acknowledgements}

Not applicable.

\section{Authors' contributions}

YY and TT conceived the study, AJ, XS, and HZ participated in the literature search, study design, data collection, data analysis, and wrote the manuscript. $\mathrm{NL}, \mathrm{SC}, \mathrm{HG}$, and MR participated in data collection and analysis and provided critical revision. ZR and XL proposed the study and participated in its design.
$\mathrm{XZ}$ and XF participated in the study design and helped with data analysis. All authors read and approved the final manuscript.

\section{Funding}

This research did not receive any specific grant from funding agencies in the public, commercial, or not-for-profit sectors.

\section{Availability of data and materials}

The data can be available upon reasonable request to the corresponding authors after and approval from the First Affiliated Hospital of Xi'an Jiaotong University Committee of Ethics.

\section{Declarations}

\section{Ethics approval and consent to participate}

The ethics committee of the First Affiliated Hospital of Xi'an Jiaotong University approved this study (No: XJTU1AF2020LSK-049). Waiving of informed consent was obtained due to the retrospective noninterventional study design.

\section{Consent for publication \\ Not applicable.}

\section{Competing interests}

The authors declare that they have no competing interests.

\section{Author details}

${ }^{1}$ Department of Medical Oncology, The First Affiliated Hospital of Xi'an Jiaotong University, No. 277 Yanta West Road, Xi'an 710061, Shaanxi, People's Republic of China. ${ }^{2}$ School of Public Health, Xi'an Jiaotong University Health Science Center, No. 76 Yanta West Road, Xi'an 710061, Shaanxi, People's Republic of China. ${ }^{3}$ Department of Hematology, The First Affiliated Hospital of Xi'an Jiaotong University, No. 277 Yanta West Road, Xi'an 710061, Shaanxi, People's Republic of China.

Received: 24 July 2021 Accepted: 27 January 2022

Published online: 07 February 2022

\section{References}

1. Sung H, Ferlay J, Siegel RL, Laversanne M, Soerjomataram I, Jemal A, et al. Global cancer statistics 2020: GLOBOCAN estimates of incidence and mortality worldwide for 36 cancers in 185 countries. CA Cancer J Clin. 2021;71(3):209-49.

2. Gudiol C, Aguado JM, Carratala J. Bloodstream infections in patients with solid tumors. Virulence. 2016;7(3):298-308.

3. Zembower TR. Epidemiology of infections in cancer patients. In: Stosor V, Zembower TR, editors. Infectious complications in cancer patients. Cham: Springer International Publishing; 2014. p. 43-89.

4. Brand JS, Colzani E, Johansson ALV, Giesecke J, Clements M, Bergh J, et al. Infection-related hospitalizations in breast cancer patients: risk and impact on prognosis. J Infect. 2016;72(6):650-8.

5. Ashour HM, El-Sharif A. Species distribution and antimicrobial susceptibility of gram-negative aerobic bacteria in hospitalized cancer patients. J Transl Med. 2009;7:14

6. Fentie A, Wondimeneh Y, Balcha A, Amsalu A, Adankie BT. Bacterial profile, antibiotic resistance pattern and associated factors among cancer patients at University of Gondar Hospital. Northwest Ethiopia Infect Drug Resist. 2018;11:2169-78.

7. Jean SS, Teng L, Hsueh PR, Ho SW, Luh KT. Antimicrobial susceptibilities among clinical isolates of extended-spectrum cephalosporin-resistant Gram-negative bacteria in a Taiwanese University Hospital. J Antimicrob Chemother. 2002;49(1):69-76.

8. Crowe MJ, Cooke EM. Review of case definitions for nosocomial infection-towards a consensus. J Hosp Infect. 1998;39(1):3-11.

9. de la Varga-Martínez O, Gómez-Sánchez E, Muñoz MF, Lorenzo M, Gómez-Pesquera E, Poves-Álvarez R, et al. Impact of nosocomial infections on patient mortality following cardiac surgery. J Clin Anesthesia. 2021;69:110104. 
10. Friedman ND, Kaye KS, Stout JE, McGarry SA, Trivette SL, Briggs JP, et al. Health care-associated bloodstream infections in adults: a reason to change the accepted definition of community-acquired infections. Ann Intern Med. 2002;137(10):791-7.

11. Chen CY, Tien FM, Sheng WH, Huang SY, Yao M, Tang JL, et al. Clinical and microbiological characteristics of bloodstream infections among patients with haematological malignancies with and without neutropenia at a medical centre in northern Taiwan, 2008-2013. Int J Antimicrob Agents. 2017:49(3):272-81.

12. Shankar-Hari M, Phillips GS, Levy ML, Seymour CW, Liu VX, Deutschman $\mathrm{CS}$, et al. Developing a new definition and assessing new clinical criteria for septic shock: for the third international consensus definitions for sepsis and septic shock (Sepsis-3). JAMA. 2016;315(8):775-87.

13. Freifeld AG, Bow EJ, Sepkowitz KA, Boeckh MJ, Ito JI, Mullen CA, et al. Clinical practice guideline for the use of antimicrobial agents in neutropenic patients with cancer: 2010 Update by the Infectious Diseases Society of America. Clin Infect Dis. 2011;52(4):427-31.

14. Mermel LA, Allon M, Bouza E, Craven DE, Flynn P, O'Grady NP, et al. Clinical practice guidelines for the diagnosis and management of intravascular catheter-related infection: 2009 Update by the Infectious Diseases Society of America. Clin Infect Dis. 2009;49(1):1-45.

15. Elbadawi HS, Elhag KM, Mahgoub E, Altayb HN, Ntoumi F, Elton L, et al. Detection and characterization of carbapenem resistant Gram-negative bacilli isolates recovered from hospitalized patients at Soba University Hospital, Sudan. BMC Microbiol. 2021;21(1):136.

16. Dimomicronpoulos G, Rovina N, Patrani M, Antoniadou E, Konstantonis D, Vryza K, et al. Past history of stage I/II solid tumor malignancy impacts considerably on sepsis mortality: a propensity score matching analysis from the hellenic sepsis study group. BMC Infect Dis. 2019;19(1):831.

17. Kamboj M, Sepkowitz KA. Nosocomial infections in patients with cancer. Lancet Oncol. 2009;10(6):589-97.

18. Nurain AM, Bilal NE, Ibrahim ME. The frequency and antimicrobial resistance patterns of nosocomial pathogens recovered from cancer patients and hospital environments. Asian Pac J Trop Biomed. 2015;5(12):1055-9.

19. Garg VK, Mishra S, Gupta N, Garg R, Sachidanand B, Vinod K, et al. Microbial and antibiotic susceptibility profile among isolates of clinical samples of cancer patients admitted in the intensive care unit at regional tertiary care cancer center: a retrospective observational study. Indian J Crit Care Med. 2019;23(2):67-72.

20. Antonio M, Gudiol C, Royo-Cebrecos C, Grillo S, Ardanuy C, Carratalà J. Current etiology, clinical features and outcomes of bacteremia in older patients with solid tumors. J Geriatric Oncol. 2019;10(2):246-51.

21. Huang CC, Wu CJ, Wang LR, Lee HC, Chang CM, Lee NY, et al. Antimicrobial susceptibility of bacteremic isolates from cancer patients with or without neutropenia at a medical center in southern Taiwan. J Microbiol Immunol Infect. 2011:44(5):376-81.

22. Schelenz S, Nwaka D, Hunter PR. Longitudinal surveillance of bacteraemia in haematology and oncology patients at a UK cancer centre and the impact of ciprofloxacin use on antimicrobial resistance. J Antimicrob Chemother. 2013;68(6):1431-8.

23. Calik Basaran N, Karaagaoglu E, Hascelik G, Durusu Tanriover M, Akova M. Prospective evaluation of infection episodes in cancer patients in a tertiary care academic center: microbiological features and risk factors for mortality. Turk J Haematol. 2016;33(4):311-9.

24. Wisplinghoff $\mathrm{H}$, Seifert $\mathrm{H}$, Wenzel RP, Edmond MB. Current trends in the epidemiology of nosocomial bloodstream infections in patients with hematological malignancies and solid neoplasms in hospitals in the United States. Clin Infect Dis. 2003;36(9):1103-10.

25. Perdikouri EIA, Arvaniti K, Lathyris D, Apostolidou Kiouti F, Siskou E, Haidich $A B$, et al. Infections due to multidrug-resistant bacteria in oncological patients: insights from a five-year epidemiological and clinical analysis. Microorganisms. 2019;7(9).

26. Gudiol C, Tubau F, Calatayud L, Garcia-Vidal C, Cisnal M, Sanchez-Ortega I, et al. Bacteraemia due to multidrug-resistant Gram-negative bacilli in cancer patients: risk factors, antibiotic therapy and outcomes. J Antimicrob Chemother. 2011;66(3):657-63.

27. Zhang Q, Gao HY, Li D, Li Z, Qi SS, Zheng S, et al. Clinical outcome of Escherichia coli bloodstream infection in cancer patients with/without biofilm formation: a single-center retrospective study. Infect Drug Resist. 2019;12:359-71.
28. Marin M, Gudiol C, Garcia-Vidal C, Ardanuy C, Carratala J. Bloodstream infections in patients with solid tumors: epidemiology, antibiotic therapy, and outcomes in 528 episodes in a single cancer center. Medicine (Baltimore). 2014;93(3):143-9.

29. Tu RH, Lin JX, Desiderio J, Li P, Xie JW, Wang JB, et al. Does intra-abdominal infection after curative gastrectomy affect patients'long-term prognosis? A multi-center study based on a large sample size. Surg Infect (Larchmt). 2019;20(4):271-7.

30. Ha YE, Kang Cl, Cha MK, Park SY, Wi YM, Chung DR, et al. Epidemiology and clinical outcomes of bloodstream infections caused by extendedspectrum $\beta$-lactamase-producing Escherichia coli in patients with cancer. Int J Antimicrob Agents. 2013;42(5):403-9.

31. Palacios-Baena ZR, Gutiérrez-Gutiérrez B, De Cueto M, Viale P, Venditti M, Hernández-Torres A, et al. Development and validation of the INCREMENT-ESBL predictive score for mortality in patients with bloodstream infections due to extended-spectrum- $\beta$-lactamase-producing Enterobacteriaceae. J Antimicrob Chemother. 2017;72(3):906-13.

32. Palacios-Baena ZR, Gutierrez-Gutierrez B, De Cueto M, Viale P, Venditti M, Hernandez-Torres A, et al. Development and validation of the INCREMENT-ESBL predictive score for mortality in patients with bloodstream infections due to extended-spectrum-beta-lactamase-producing Enterobacteriaceae. J Antimicrob Chemother. 2017;72(3):906-13.

33. Soeters PB, Wolfe RR, Shenkin A. Hypoalbuminemia: pathogenesis and clinical significance. JPEN J Parenter Enteral Nutr. 2019;43(2):181-93.

34. Gupta D, Lis CG. Pretreatment serum albumin as a predictor of cancer survival: a systematic review of the epidemiological literature. Nutr J. 2010;9:69.

35. Paccagnella A, Morassutti I, Rosti G. Nutritional intervention for improving treatment tolerance in cancer patients. Curr Opin Oncol. 2011;23(4):322-30.

36. Fruchtenicht AVG, Poziomyck AK, Kabke GB, Loss SH, Antoniazzi JL, Steemburgo T, et al. Nutritional risk assessment in critically ill cancer patients: systematic review. Rev Bras Ter Intensiva. 2015;27(3):274-83.

\section{Publisher's Note}

Springer Nature remains neutral with regard to jurisdictional claims in published maps and institutional affiliations.

Ready to submit your research? Choose BMC and benefit from:

- fast, convenient online submission

- thorough peer review by experienced researchers in your field

- rapid publication on acceptance

- support for research data, including large and complex data types

- gold Open Access which fosters wider collaboration and increased citations

- maximum visibility for your research: over 100M website views per year

At BMC, research is always in progress.

Learn more biomedcentral.com/submissions 